\title{
Metaphysics of science between metaphysics and science
}

\author{
Michael Esfeld \\ University of Lausanne, Department of Philosophy \& \\ Centre romand for logic, history and philosophy of science \\ CH-1015 Lausanne, Switzerland \\ Michael-Andreas.Esfeld@unil.ch
}

(forthcoming in Grazer Philosophische Studien 74 (2007), pp. 199-213)

\begin{abstract}
The paper argues that metaphysics depends upon science when it comes to claims about the constitution of the real world. That thesis is illustrated by considering the examples of global supervenience, the tenseless vs. the tensed theory of time and existence, events vs. substances, and relations vs. intrinsic properties. An argument is sketched out for a metaphysics of a fourdimensional block universe whose content are events and their sequences, events consisting in physical properties instantiated at space-time points, these properties being relations rather than intrinsic properties.
\end{abstract}

\section{Introduction}

Metaphysics used to be and again is the core discipline of philosophy. In the words of Frank Jackson,

Metaphysics ... is about what there is and what it is like. But of course it is concerned not with any old shopping list of what there is and what it is like. Metaphysicians seek a comprehensive account of some subject matter - the mind, the semantic, or, most ambitiously, everything - in terms of a limited number of more or less basic notions. In doing this they are following the good example of physicists. The methodology is not that of letting a thousand flowers bloom but rather that of making do with as a meagre a diet as possible. (Jackson 1994, 25)

Metaphysics, thus conceived, seeks a comprehensive account of what there is in the real world. How do we gain knowledge about the world? When it comes to explaining how the manifest phenomena are connected, science tells us what there is in the world. As Wilfrid Sellars once put it, "in the dimension of describing and explaining the world, science is the measure of all things, of what is that it is, and of what is not that it is not" ("Empiricism and the Philosophy of Mind" (1956) in Sellars 1963, 173). There is no source of philosophical knowledge about the world independent of science. In seeking a comprehensive account of everything, metaphysics is continuous with science, going beyond particular scientific theories. The thesis of this paper is that there is a mutual dependence between science and philosophy: philosophy in the sense of metaphysics needs science to know about what there is in the real world, and science needs philosophy in the sense of epistemology when it comes to developing criteria for the interpretation of scientific theories - that is, criteria for the assessment of knowledge claims contained in scientific theories.

It is common to distinguish between the epistemology and the metaphysics of science. David Papineau, for one, draws this distinction in the following way: 
The philosophy of science can usefully be divided into two broad areas. The epistemology of science deals with the justification of claims to scientific knowledge. The metaphysics of science investigates philosophically puzzling features of the world described by science. In effect, the epistemology of science asks whether scientific theories are true, whereas the metaphysics of science considers what it would tell us about the world if they were. (Papineau 1996, 1)

Whereas the philosophy of science was dominated for decades by epistemological issues under the influence of logical empiricism and its critics, the metaphysics of science has gathered momentum in the last two decades or so. The type of metaphysics that is at issue is a revisionary in contrast to a descriptive metaphysics, to use Peter Strawson's terms (1959, introduction). The justification for a revisionary metaphysics stems from science: our best scientific theories suggest the conclusion that a number of our common sense beliefs about the constituents of the world - as analysed by what Strawson calls descriptive metaphysics are false.

In the following, I shall sketch out four examples that illustrate the interplay between science and metaphysics - examples that show how scientific results provide a content for metaphysics, namely a content that results in a revisionary metaphysics. The examples are (1) global supervenience, (2) tenseless vs. tensed theories of time and existence, (3) events vs. substances, and (4) relations vs. intrinsic properties. In conclusion, I shall sum up the resulting view of the fundamental ingredients of the world and mention the most important open issues.

\section{Example 1: global supervenience}

When metaphysics seeks a comprehensive account of everything in terms of a limited number of basic notions, some entities have to be considered as being fundamental and all the other ones as being dependent on those fundamental entities. It is common to spell that idea out in terms of global supervenience: there is a fundamental level of the world, and everything else supervenes on what there is on that level. To quote again Frank Jackson, "Any world which is a minimal physical duplicate of our world is a duplicate simpliciter of our world" (1998, 8). A minimal physical duplicate of our world can be taken to be what one gets if one duplicates the fundamental physical level of our world. Although this is a thesis of logical supervenience, it is contingent that the actual world is a world in which supervenience holds. Hence, the thesis of global supervenience with respect to the actual world cannot be established on the basis of philosophical reflection alone, but it is based upon knowledge of our world, knowledge that originates in science.

Why should one accept global supervenience? We have physical theories at our disposal that are both fundamental and universal - namely, at the current state of physics, quantum field theory and general relativity. These theories are universal because they apply to everything that there is in the natural world. Everything is a physical system in the sense that it is subject to the laws of gravitation (general relativity) and electromagnetism (quantum field theory), among others. There are some systems to which only these theories apply. Let us call the level of these systems the fundamental level of the world. Let us furthermore assume that this level consists in the distribution of physical properties at space-time points over the whole of space-time. There is nothing smaller than a point. A field is commonly defined over the whole of space-time, with field properties being attributed to the points and regions of space-time. 
By contrast, chemical or biological theories, for instance, are not fundamental. It is not the case that everything that there is in the world is a chemical system (such as a molecule) or a biological system (such as an organism). The mentioned physical theories are fundamental with respect to all the other current theories of science in the sense that these theories never need to have recourse to concepts, laws and explanations from any other theories, whereas all our other theories sometimes need to have recourse to concepts, laws and explanations from general relativity or quantum field theory. Chemical or biological theories sometimes have to invoke physical concepts and laws that are in the last resort concepts and laws of general relativity or quantum field theory - for instance, in order to explain why a given chemical or biological regularity has an exception in a particular situation. If there are laws in chemistry or biology, these are ceteris paribus laws, whereas the laws of fundamental physics are strict laws, admitting no exceptions.

One can sum up these considerations by putting forward a principle of the causal, nomological and explanatory completeness of the fundamental level: for any fundamental physical system $\mathrm{p}$ (i.e. instantiation of physical properties at a space-time point), insofar as $\mathrm{p}$ has causes, comes under laws and admits of explanations, there are causes that are only fundamental physical causes, there are laws that are only fundamental physical laws and there are explanations that employ only concepts of fundamental physics. This principle does not exclude that for any fundamental physical system $p$, there are other causes, laws, or explanations. But such other causes, laws, or explanations, if they exist, do not contribute anything that is not contributed by fundamental physical laws, causes, and explanations.

The principle of completeness does not occur within physics. It is a principle of the metaphysics of science, belonging to a reasonable interpretation of what our fundamental physical theories tell us about the world (for an extensive argument, see Papineau 2002, appendix). If one did not endorse that principle, an unpalatable consequence would ensue: one would be committed to saying that our fundamental physical theories are either inapplicable to some phenomena in their domain (i.e. physical properties being instantiated at space-time points), because these phenomena are covered by causes, laws and explanations of a higherlevel theory instead of fundamental physical causes, laws and explanations; or, if applicable, these theories are false, because there are some phenomena in their domain for which the predictions in terms of causes, laws and explanations of these theories yield the wrong results, these phenomena being under the influence of causes, laws and explanations of a higher-level theory instead (the recent criticism of the principle of completeness that Bishop 2006 voices comes down to exploring that possibility).

To illustrate that point, consider the example of mental causes and brain states - putting in brain states for fundamental physical phenomena, and mental causes for higher-level causes that are distinct from physical causes. Assume that there are mental causes that are not neurobiological causes and that bring about neurobiological effects that are caused only by them, say produce certain brain states. In that case, as regards the brain states in question, the relevant neurobiological theory would be either inapplicable, because these states are subject to the influence of certain non-biological, mental causes; or it would be false, yielding the wrong probabilities for the occurrence of the brain states in question. If, for instance, the occurrences of a mental intention of the type raising one's right arm were distinct from neurobiological states and if these occurrences of mental intentions produced neurobiological effects that are not produced by neurobiological causes, then any mental intention of that type 
would regularly raise the probability for brain states of certain types to occur - a probability that would be different from the neurobiological probability that takes only biological factors into account. However, there is not the slightest evidence that the physical or neurobiological laws break down in one of these ways in some area of the brain when the mental is present. Thus, in short, as far as the principle of completeness is concerned, the argument is a philosophical one (as with any claim in the metaphysics of science) - but if the argument were not true, a consequence unacceptable for science would ensue.

The principle of completeness does not imply global supervenience. It rules out causes, laws and explanations that contribute something to fundamental physical phenomena that is not provided by fundamental physical causes, laws and explanations; but it does not exclude that there are emergent epiphenomena in our world that would not necessarily be duplicated if one created a duplicate of the fundamental physical level of our world. However, admitting such emergent epiphenomena would again lead to consequences that are unacceptable to science: such emergent epiphenomena would be such that it would in principle be impossible to find any explanation for them.

In order to strengthen that point, one can draw on another principle, namely the principle of evolution: everything that there is in the real world apart from fundamental physical systems developed out of fundamental physical systems. Given evolution, making a duplicate of the fundamental physical level of the real world would amount to duplicating cosmic evolution. If anything that there is in the real world were missing in the duplicate, everything that we know from science would lead us to expect that there also is some difference in the distribution of fundamental physical properties in that duplicate with respect to the real world - difference in some spontaneous mutation, for instance (and there is no such biochemical difference without there also being a fundamental physical difference). Consequently, such a possible world would after all not be an exact duplicate of the fundamental physical level of the real world. Completeness, if conjoined with evolution, therefore provides a strong reason for endorsing global supervenience.

Note that, according to global supervenience, what there is on the fundamental level determines everything that there is in the real world, but that the issue of determinism is a different matter. Global supervenience considers the distribution of the fundamental physical properties over the whole of space-time as the supervenience base. Determinism concerns the question of whether or not the evolution in time is deterministic. If global supervenience is valid and if the real world is deterministic, having, say, the big bang as initial condition, then any duplicate of the big bang and the laws of nature would be sufficient to amount to a duplicate of cosmic evolution. But this is a much stronger thesis than global supervenience. Global supervenience says something about worlds that are a physical duplicate of our world - that is, a duplicate of the distribution of fundamental physical properties over the whole of space-time -, independently of whether or not there is physical determinism. If there is quantum indeterminism and, say, two possible worlds $w_{1}$ and $w_{2}$ agree until a certain time $t$ in the distribution of the fundamental properties and then diverge because of one radioactive atom decaying in $w_{1}$ but not in $w_{2}$, then $w_{2}$ is not a minimal physical duplicate of $w_{1}$.

\section{Example 2: the tenseless vs. the tensed theory of time and existence}

Let us have a closer look at the fundamental level, the distribution of physical properties at space-time points over the whole of space-time. There are two rival theories of time as well as 
of existence. According to the tensed theory of existence, existence is relative to a time in the sense that only that what is present - or only that what is present and what is past - exists. What is in the future does not exist as yet, and, according to some versions of this theory, what is past does not exist any more. The tensed theory of existence implies the tensed theory of time according to which there is a flow of time; the past, the present and the future are objective modes of time, being out there in the world. The tensed theory of time, however, does not imply the tensed theory of existence.

Opposed to the tensed theory of time is the tenseless theory of time which claims that there are only temporal relations of being earlier than, simultaneous with and later than among events, but no objective modes of past, present and future. The tenseless theory of time implies the tenseless theory of existence according to which existence is not relative to a location in time in the same way as it is not relative to a location in space: everything that there is in space and time simply exists. The tenseless theory of existence, however, does not imply the tenseless theory of time.

There are philosophical arguments in favour of both these theories of time and existence. The case can be settled by taking science into account. The relevant scientific theory is special relativity. Special relativity shows that there is no objective simultaneity. Any event in the sense of physical properties occurring at a space-time point - that is supposed to be simultaneous with other events is so only relative to a reference frame, and there is no globally preferred reference frame. Thus, there is no objective "now" - in the same way as there is no objective "here". For any space-time point, it can be claimed that it is "present" in the same way as it can be claimed that it is "here" (see, for instance, Dorato 1995, chapters 11 to 13 , in particular pp. 186-187, 210). The reason is that, according to special relativity, spatial as well as temporal distances between events are relative to a reference frame. Invariant with respect to the choice of a reference frame is only the four-dimensional, spatiotemporal distance between any two events (or points of space-time). That is the reason why special relativity is taken to show that space and time are united in a four-dimensional entity, space-time. General relativity - and notably its application in cosmology - goes beyond special relativity; but it does not change anything with respect to what special relativity says about the relativity of spatial and temporal distances.

Special relativity hence makes a case for the tenseless theories of time and existence. Since spatial and temporal distances are relative to a reference frame, there is no basis in the physical world for upholding a tensed theory of time or existence (see Saunders 2002). Again, this claim belongs to the metaphysics of science. It is logically possible to rescue the idea of an objective present by introducing the notion of one globally privileged reference frame. That notion does not contradict special relativity. The point is that it is entirely ad hoc - so that, rejecting the tenseless theories of time and existence as a claim of the metaphysics of science again has consequences that are unacceptable for science.

\section{Example 3: events vs. substances}

The tenseless theories of time and existence, based on the physics of special relativity, result in what is known as the view of the world as a block universe: the whole of four-dimensional space-time is a single block so to speak, including time; everything that there is exists at a space-time point or region. What is the content of the block universe? 
In metaphysics, it is common to draw a distinction between substances and events. Substances persist as a whole for a certain time. They have spatial parts (unless they are atoms in a literal sense), but they do not have any temporal parts. Relying on physics, an event can be conceived as the physical properties instantiated at a space-time point. Continuous sequences of events are processes. Processes have spatial as well as temporal parts. Four-dimensional entities such as processes are commonly conceived as perdurants, persisting by having spatial as well as temporal parts, whereas three-dimensional entities such as substances are conceived as endurants, persisting as a whole for a certain time, having no temporal parts.

Common sense admits both substances and processes. A volcano, for instance, is regarded as a substance, persisting for a certain time by having no temporal parts, but only spatial parts. The eruption of a volcano, by contrast, is a process, persisting for a time by having temporal as well as spatial parts. The eruption can, for instance, be first mild and then heavy.

Events and processes cannot be dispensed with in metaphysics. Even if it were possible to conceive all events as consisting in changes of the properties of substances, there would be a dualism of substances and events qua changes in the properties of substances. However, it may be possible to do without substances, recognizing only events and processes. (There is an ambiguity in the notion of a substance: If one regards space-time points as substances, they are not substances in the sense of endurants, but four-dimensional entities that have neither spatial nor temporal parts; they are not in space and time, but they are what makes up spacetime). Again, there is a philosophical dispute as to whether or not one should admit substances in addition to events. Again, science is relevant to that dispute.

If one switches from a physics of three-dimensional space to a physics of four-dimensional space-time (block universe), there no longer is any need to admit substances as the entities that are the enduring foundation of change, change being the change of properties of substances, motion being change in the location of substances. Moreover, there is no need to conceive the identity of things as the identity of substances in time, because substances do not have temporal parts.

In the metaphysics of the block universe, identity can be accounted for in terms of genidentity, that is, sequences of events that instantiate the same or similar properties. In other words, the identity of any physical object in time is explained by the fact that the object is a process whose temporal parts form a continuous sequence, exhibiting similar physical properties. As regards motion, what common sense considers as the motion of a substance through three-dimensional space is explained as a continuous sequence of space-time points or regions that possess a similar physical content (a world line). Change is different physical properties instantiated at points or regions of space-time forming a continuous sequence.

Hence, given the physics of special relativity, the arguments for a metaphysics of science that is a metaphysics of events - and processes (perdurants) -, admitting no substances (endurants) are in the first place the philosophical ones of coherence and parsimony: if one makes the step to a metaphysics of a four-dimensional block universe, it is simply not coherent to recognize three-dimensional substances among the content of the block universe. Four-dimensional events and their sequences (processes) have to be accepted anyway, and they are sufficient as the furniture of the universe (see Sider 2001 as regards the philosophical arguments). 
Moreover, in recent years, arguments have been developed to the effect that admitting three-dimensional substances with spatial, but no temporal parts is not consistent with special relativity. According to special relativity, the spatial distances between points depend on a reference frame. Consequently, if one subscribes to an ontology according to which there are three-dimensional macroscopic substances, their spatial figure varies from one frame of reference to another one, because the spatial distances between the points that the substance in question occupies depend on a reference frame. If, by contrast, physical objects are fourdimensional perdurants, their figure in four-dimensional space-time is not relative to a reference frame (see Balashov 1999 as well as Hales \& Johnson 2003). A further argument makes the following point: since simultaneity is relative to a reference frame, a metaphysics of enduring three-dimensional objects cannot come up with a convincing theory of the coexistence (copresence) of objects. By contrast, a metaphysics of perduring fourdimensional objects, which have temporal parts, can easily include a theory of coexistence: any two four-dimensional objects coexist if and only if they have parts that are separated by a space-like interval (see Balashov 2000 and the discussion between Gilmore 2002 and Balashov 2005). If these arguments prove sound, they put the case against three-dimensional substances on a par with the case against objective simultaneity based on special relativity.

\section{Example 4: relations vs. intrinsic properties}

Up to now, I have argued in favour of the view of the basic level of the world consisting in the distribution of fundamental physical properties at space-time points over the whole of space-time, forming continuous sequences that are processes and that can be regarded as physical objects (albeit no substances in the sense of things that do not have temporal parts). Quantum physics can be seen in the first place as adding something to this view concerning the physical properties. Whereas special and general relativity can be conceived as theories about space-time notably, quantum physics is concerned with matter.

It is often taken for granted that the fundamental physical properties, instantiated at spacetime points, are intrinsic properties. Intrinsic are all and only those properties that an object has irrespective of whether or not there are other contingent objects; in brief, having or lacking an intrinsic property is independent of accompaniment or loneliness (see Langton \& Lewis 1998 and for a refinement Lewis 2001). All other properties are extrinsic or relational, consisting in the object bearing certain relations to other objects.

Quantum physics is usually conceived in terms of states of physical systems. The state of a system at a time can be regarded as encapsulating the properties that the system has at that time. The most striking feature of quantum theory is that the states of several systems can be entangled. In fact, starting from the formalism of quantum theory, it is to be expected that whenever one considers a complex system that consists of two or more quantum systems, the states of these systems are entangled. Entanglement is to say that it is not the case that each of the systems has a state separately. On the contrary, only the whole, that is, the complex system composed of two or more systems, is in a precise state (called a "pure state"). Philosophers of physics therefore speak of non-separability (Howard 1989) or relational holism (Teller 1986), since entanglement consists in certain relations among quantum systems. These relations give rise to correlations that are confirmed by experiments.

These relations cannot be traced back to intrinsic properties of the physical systems in question. There are no intrinsic properties of the related quantum systems on which the 
relations of entanglement could supervene. Quantum physics can therefore be taken to suggest a metaphysics of relations, known also as structural realism: insofar as quantum physics is concerned, the fundamental physical properties consist in certain relations instead of being intrinsic properties (see French \& Ladyman 2003 and Esfeld 2004).

Again, this is a conclusion belonging to the metaphysics of science. This conclusion could be avoided by postulating intrinsic properties in the form of hidden variables that restore separability among quantum systems (that is, properties of quantum systems that are there, but whose value we cannot know). However, since the discovery of the theorem of John Bell (1964), it is clear that one would have to pay a high metaphysical as well as physical price for admitting hidden variables of that kind. Again, if one does not accept this position in the metaphysics of science - i.e., a metaphysics of relations as fundamental physical properties, not supervening on intrinsic properties -, one faces consequences that are not acceptable to science, namely being committed to hidden variables that are intrinsic properties. (The only elaborate account of quantum physics in terms of hidden variables, Bohm's theory, does not fall within the scope of that criticism, for Bohm himself interprets his theory rather in terms of relations and holism than in terms of intrinsic properties; see Bohm \& Hiley 1993).

\section{Conclusion}

Let us take stock. The examples discussed in the preceding sections suggest a view of the fundamental level of the world according to which the world is a four-dimensional block universe whose content are events and their sequences, events consisting in physical properties instantiated at space-time points, these properties being - as far as quantum physics is concerned - relations rather than intrinsic properties. This certainly is a revisionary metaphysics, rejecting a tensed view of time and existence, admitting only events and processes instead of substances (endurants) and giving priority to relations instead of intrinsic properties. Everything else there is in the world supervenes on that fundamental level in the sense of the mentioned thesis of global supervenience. The rationale for this metaphysics stems from science. The claims sketched out in the preceding sections are a reasonable interpretation of what science tells us about the world. Not endorsing them would lead to consequences that are unacceptable for science.

Nonetheless, there are a number of open issues skipped in the preceding sections:

- the relationship between space-time and matter: In the preceding sections, I have used the terminology of physical properties being instantiated at space-time points or regions. Are the physical properties literally properties of space-time points or regions - so that matter reduces to properties of space-time? Or is there a dualism between space-time and matter fields being inserted in space-time? This is an open issue in the philosophy of general relativity in the first place. On the one hand, the project of an outright reduction of matter to space-time failed. (This is the project of Wheeler's geometrodynamics; see Wheeler 1962 and for the acknowledgement of its failure Misner, Thorne \& Wheeler 1973, § 44.3-4, in particular p. 1205). On the other hand, there is no clear distinction between space-time and matter in general relativity: the metric field includes spatio-temporal properties, such as the spatio-temporal distances between space-time points, as well as material properties, namely gravitation. However, resolving this issue depends not only on the philosophy of general relativity, but also on the open issue of the unification of general relativity and quantum field theory. 
- the unification of general relativity and quantum field theory: For the time being, there are two fundamental physical theories: quantum field theory and general relativity. The relationship between these two theories is not clear. It is desirable to have one fundamental physical theory. If the project of unifying quantum field theory and general relativity succeeds, the resulting scientific theory may have important repercussions for our view of the basic level of nature. To be more precise without engaging in as yet premature speculations, quantum entanglement is independent of the spatio-temporal distance of the quantum systems whose states are entangled. This may be taken as one hint among others that space-time points are not the most fundamental level of nature. The level of physical properties instantiated at space-time points is, of course, fundamental with respect to all the other known levels - such as the levels of chemical, biological properties, etc. But there may be a quantum level that is more fundamental than the level of space-time points, space-time being somehow derived from that quantum level. If such a view were to prove sound in the future, it would have important implications for the metaphysics of the physical world (and, perhaps, the definition of "physical" itself). Nonetheless, whatever may be the future fundamental physical theory that achieves a unified treatment of the phenomena that are currently considered by two different theories, it would be unreasonable to expect that future theory to go back behind the unification of space and time as considered by general relativity or the holism that quantum entanglement manifests. Even if we ignore as yet the content of that future theory, the metaphysical direction seems clear: events instead of enduring substances, and relations instead of intrinsic properties.

- the micro-macro relationship: This is what the famous measurement problem in the interpretation of quantum physics is about. That problem is still unsolved. The point at issue is the extension of quantum entanglement. Is there a physical process that leads to the dissolution of quantum entanglement so that there really are macroscopic systems having well-defined properties separately - such as cats being always either alive or dead, their states not being entangled with the states of other systems (cf. the famous thought experiment of Schrödinger's cat (Schrödinger 1935, 812)? There is a physical proposal for a further development of the formalism of quantum theory in that sense, going back to Ghirardi, Rimini \& Weber (1986). But that proposal faces a number of physical problems. To my mind, this issue is rather an open physical one than a metaphysical one.

These open issues show that the metaphysics of science is an unfinished business. The metaphysics of science depends on science and its progress. In a nutshell, the metaphysics of science is as hypothetical as is science. However, since there is no source of philosophical knowledge about the constitution of the world that is independent of science, this is all that can be achieved in a metaphysics of the real world - and it is sufficient to turn the metaphysics of science into an exciting business, worth engaging in.

\section{References}

Balashov, Yuri (1999): "Relativistic objects”. Noûs 33, pp. 644-662.

Balashov, Yuri (2000): "Enduring and perduring objects in Minkowski space-time”. Philosophical Studies 99, pp. 129-166.

Balashov, Yuri (2005): "Special relativity, coexistence and temporal parts: a reply to Gilmore”. Philosophical Studies 124, pp. 1-40.

Bell, John S. (1964): “On the Einstein-Podolsky-Rosen-paradox”. Physics 1, pp. 195-200. 
Bishop, Robert C. (2006): “The hidden premiss in the causal argument for physicalism”. Analysis 66, pp. 44-52.

Bohm, David \& Hiley, Basil (1993): The undivided universe. An ontological interpretation of quantum theory. London: Routledge.

Dorato, Mauro (1995): Time and reality. Spacetime physics and the objectivity of temporal becoming. Bologna: Cooperativa Libraria Universitaria Editrice Bologna.

Esfeld, Michael (2004): "Quantum entanglement and a metaphysics of relations". Studies in History and Philosophy of Modern Physics 35B, pp. 601-617.

French, Steven \& Ladyman, James (2003): "Remodelling structural realism: quantum physics and the metaphysics of structure". Synthese 136, pp. 31-56.

Ghirardi, Giancarlo, Rimini, Alberto \& Weber, Tullio (1986): “Unified dynamics for microscopic and macroscopic systems”. Physical Review D34, pp. 470-491.

Gilmore, Cody S. (2002): "Balashov on special relativity and temporal parts". Philosophical Studies 109, pp. 241-263.

Hales, Steven D. \& Johnson, Timothy A. (2003): "Endurantism, perdurantism, and special relativity". Philosophical Quarterly 53, pp. 524-539.

Howard, Don (1989): "Holism, separability, and the metaphysical implications of the Bell experiments". In: J. T. Cushing \& E. McMullin (eds.): Philosophical consequences of quantum theory. Reflections on Bell's theorem. Notre Dame: University of Notre Dame Press. Pp. 224-253.

Jackson, Frank (1994): “Armchair metaphysics”. In: J. O’Leary-Hawthorne \& M. Michael (eds.): Philosophy in mind. Dordrecht: Kluwer. Pp. 23-42. Reprinted in Frank Jackson (1998): Mind, method and conditionals. Selected essays. London: Routledge. Pp. 154-176.

Jackson, Frank (1998): From metaphysics to ethics. A defence of conceptual analysis. Oxford: Oxford University Press.

Langton, Rae \& Lewis, David (1998): "Defining 'intrinsic'”. Philosophy and Phenomenological Research 58, pp. 333-345. Reprinted in David Lewis (1999): Papers in metaphysics and epistemology. Cambridge: Cambridge University Press. Pp. 116-132.

Lewis, David (2001): “Redefining “intrinsic”. Philosophy and Phenomenological Research 63, pp. 381-398.

Misner, Charles W., Thorne, Kip S. \& Wheeler, John A. (1973): Gravitation. San Francisco: Freeman.

Papineau, David (1996): “Introduction”. In: D. Papineau (ed.): The philosophy of science. Oxford: Oxford University Press. Pp. 1-20.

Papineau, David (2002): Thinking about consciousness. Oxford: Oxford University Press.

Saunders, Simon (2002): "How relativity contradicts presentism". In: C. Callender (ed.): Time, reality \& experience. Cambridge: Cambridge University Press. Pp. 277-292.

Schrödinger, Erwin (1935): “Die gegenwärtige Situation in der Quantenmechanik". Naturwissenschaften 23, pp. 807-812, 823-828, 844-849.

Sellars, Wilfrid (1963): Science, perception and reality. London: Routledge.

Sider, Theodore R. (2001): Four-dimensionalism. Oxford: Clarendon Press.

Strawson, Peter F. (1959): Individuals. An essay in descriptive metaphysics. London: Routledge.

Teller, Paul (1986): "Relational holism and quantum mechanics". British Journal for the Philosophy of Science 37, pp. 71-81.

Wheeler, John A. (1962): Geometrodynamics. New York: Academic Press. 